\title{
TERT promoter mutations in thyroid cancer: a report from a Middle Eastern population
}

\author{
Ebtesam Qasem ${ }^{1, *}$, Avaniyapuram Kannan Murugan, ${ }^{1, *}$, Hindi Al-Hindi², \\ Mingzhao Xing ${ }^{4}$, Mai Almohanna', Meshael Alswailem ${ }^{1}$ and Ali S Alzahrani ${ }^{1,3}$ \\ 'Department of Molecular Oncology, King Faisal Specialist Hospital and Research Centre, Riyadh, Saudi Arabia \\ ${ }^{2}$ Department of Pathology and Laboratory Medicine, King Faisal Specialist Hospital and Research Centre, Riyadh, \\ Saudi Arabia \\ ${ }^{3}$ Department of Medicine, King Faisal Specialist Hospital and Research Centre, Riyadh, Saudi Arabia \\ ${ }^{4}$ Division of Endocrinology and Metabolism, Johns Hopkins University School of Medicine, Baltimore, \\ Maryland, USA \\ *(E Qasem and A K Murugan contributed equally to this work)
}

Correspondence should be addressed to A S Alzahrani Email aliz@kfshrc.edu.sa

\begin{abstract}
Telomerase reverse transcriptase (TERT) promoter mutations C228T and C250T have recently been described in follicular cell-derived thyroid cancer (TC) in patients from North America and Europe. In this study, we explored whether these findings could be replicated in patients from a different ethnic group. We screened 17 benign thyroid adenomas and 265 TC samples from patients in the Middle East for these mutations by PCR and direct sequencing using DNA isolated from paraffin-embedded tumor tissues. None of the 17 benign adenomas harbored TERT promoter mutations. Of 265 TC, 34 (12.8\%) harbored TERT promoter mutations, including 10/153 (6.5\%) conventional papillary TC (CPTC), 8/57 (14.0\%) follicular variant PTC, $9 / 30(30 \%)$ tall cell variant PTC, $1 / 3(30 \%)$ Hurthle cell thyroid cancer (HTC), $1 / 5(20 \%)$ follicular TC, and 5/13 (38.5\%) poorly differentiated TC. C250T mutation was present in only 6/265 $(2.3 \%)$ cases, while C228T mutation was present in a total of 28/265 (10.6\%) cases. These two mutations were mutually exclusive. TERT promoter mutations were significantly more common in older ( $\geq 45$ years) than younger patients and were associated with larger tumour size, vascular invasion, higher TNM stage (stage III and IV), BRAF V600E mutation and persistent/recurrent disease at 6-12 months after initial treatment and at the last follow up. These associations were stronger in non-CPTC. Thus, this study on a large cohort of TC patients from Middle East demonstrates that TERT promoter mutations are relatively common, especially in the non-CPTC, and are associated with more aggressive histopathological features, $B R A F^{V 600 E}$ mutation, and disease persistence/recurrence than the WT TERT.
\end{abstract}

Key Words

- thyroid

- carcinoma

\section{Introduction}

Differentiated thyroid cancer (DTC) is the most common endocrine malignancy (Jemal et al. 2011). Its incidence has been rapidly increasing over the last four decades (Jemal et al. 2011, 2013, Jung et al. 2014). Although the widespread use of neck ultrasonography
(US) has contributed to the discovery of an increasing number of cases of small DTC that would otherwise remain undiagnosed, some data have suggested a true increase in the incidence of this cancer (Enewold et al. 2009).

Published by Bioscientifica Ltd.
Endocrine-Related Cancer (2015) 22, 901-908 
Major progress in our understanding of the molecular pathogenesis of DTC has taken place over the last decade (Xing 2013). The contribution of genetic mutations in the MAPK and phosphatidylinositide 3-kinases (PI3KCa)-AKT pathways in the pathogenesis of DTC are well established, and new pathways and genes are being discovered as additional drivers for the initiation and progression of DTC (Agrawal et al. 2014). These new genetic aberrations include mutations in the telomerase reverse transcriptase (TERT) promoter (Landa et al. 2013, Liu et al. 2013, 2014a, Vinagre et al. 2013, Liu \& Xing 2014, Melo et al. 2014, Ngeow \& Eng 2014, Wang et al. 2014a, Xing et al. 2014, Gandolfi et al. 2015). TERT is the catalytic subunit of telomerase which is composed of a ribonucleoprotein complex that is responsible for adding telomeric fragments to chromosomes, maintaining the telomere length that usually shortens over time in the dividing somatic cells (Smekalova et al. 2012). This mechanism plays a major role in cellular immortality (Smekalova et al. 2012, Mocellin et al. 2013). The activity of TERT varies between different cells, but it is frequently up-regulated in many types of cancer cells (Smekalova et al. 2012, Mocellin et al. 2013). This seems to provide cancer cells with increasing survival advantage and contributes to their extended division and survival (Blackburn 2005). Although mutations in the coding regions of the TERT gene are rare, TERT promoter mutations (C288T and C250T) have recently been described in many types of human cancers, including the follicular cell-derived TC (Landa et al. 2013, Liu et al. 2013, Vinagre et al. 2013). They were also found to be associated with more aggressive tumors and with high rate of $B R A F^{V 600 E}$ mutation (Landa et al. 2013, Liu et al. 2013, 2014a,b, Liu \& Xing 2014, Melo et al. 2014, Wang et al. 2014a, Xing et al. 2014, Gandolfi et al. 2015, Shi et al. 2015). These studies have been mostly conducted in North American and European populations. The frequency and significance of TERT promoter mutations in follicular cell-derived TC have not been well studied in other ethnic groups. In this study, we report the prevalence and prognostic value of TERT mutations in a large series of patients with TC from the Middle East.

\section{Patients and methods}

\section{Patients}

We studied 282 surgically removed thyroid tumors obtained from 265 non-selected patients with TC and 17 patients with benign multinodular goitre seen during the period January 2008 to November 2011 at King Faisal
Specialist Hospital and Research Centre, Riyadh, Saudi Arabia. The TC patients comprised 201 females (75.8\%) and 64 males (24.2\%) with a median age of 34 years (range, 1178 years) at the initial treatment. The tumors included 153 (57.7\%) conventional papillary TCs (CPTCs), 57 (21.1\%) follicular variant PTCs (FVPTC), 30 (11.3\%) tall-cell PTCs (TC-PTC), 4 (1.5\%) diffuse sclerosing type TCs, 3 (1.1\%) Hurthle cell cancer, 5 (1.9\%) follicular TCs (FTCs), and 13 (4.9\%) poorly DTCs (PDTCs). Data were extracted from the medical and pathological reports of the patients, and the study protocol was approved by the Institutional Review Board. All patients with DTC underwent near-total or total thyroidectomy, and 227 of them (85.7\%) underwent unilateral or bilateral neck dissection. Two hundred and 43 patients (91.7\%) underwent radioactive iodine (RAI) ablation 6-12 weeks after thyroidectomy with a median dose of $122 \mathrm{mCi}$ (range, 29-210). The patients were treated with L-thyroxine suppression and followed regularly every 6-12 months. TNM staging revealed that 179 patients $(67.5 \%)$ were in stage I, $26(9.8 \%)$ in stage II, 18 (6.8\%) in stage III, and 34 (12.8\%) in stage IV. Eight (3\%) cases were non-stageable due to incomplete pathological information. Remission was defined by stimulated thyroglobulin (Tg) level of $\leq 1 \mathrm{ng} / \mathrm{dl}$ in the absence of anti-Tg autoantibodies and negative neck US and other imaging studies (when done). When $\mathrm{Tg}$ was undetectable but anti-Tg autoantibody levels were elevated, we relied on the findings of US, diagnostic radioiodine whole body scan (DxWBS) and other imaging studies. Persistence was defined by any or a combination of the following findings: a stimulated $\mathrm{Tg}$ level of $>1 \mathrm{ng} / \mathrm{dl}$, positive US-guided fine-needle aspiration (FNA), or positive radioiodine body scan when performed. Recurrence was considered similar to persistence after at least a 6-month period of remission. Patients in whom the status could not be determined due to inadequate data or elevated $\mathrm{Tg}$ levels in the presence of elevated Tg autoantibodies were excluded from the analysis of outcome.

\section{DNA isolation, PCR and direct sequencing}

The pathological specimen from each patient was examined by an experienced endocrine pathologist ( $\mathrm{H} \mathrm{A})$, and the tumor tissue was carefully dissected from formalin-fixed paraffin-embedded tissue blocks. We did not exclude patients with concomitant Hashimoto's thyroiditis or colloid goiter but the dissection was carefully performed to ensure that the specimens contain tumor tissue only with minimal non-tumor tissue. DNA was extracted using a commercial DNA extraction kit (QIAamp DNA FFPE Tissue Kit, Qiagen, Catalog No. 56404)

Published by Bioscientifica Ltd. 
according to the manufacturer's instructions. DNA was quantified using a nanodrop2000 spectrophotometer (Thermo Scientific, Willmington, DE, USA) and DNA purity was assured by the A260/280 ratio, with a ratio of $\geq 1.8$ indicating good purity. We used PCR and direct sequencing using Big Dye terminator v3.1 cycle-sequencing reaction kit and an ABI PRISM 3730Xl genetic analyzer (Applied Biosystems) to detect TERT promoter mutations using the same primers and PCR conditions described previously (Liu et al. 2013). BRAF $F^{V 600 E}$ mutation was examined by amplifying and sequencing exon 15 of the $B R A F$ gene using previously described primers and PCR conditions (Liu et al. 2013).

\section{Statistical analysis}

The Statistical Package for Social Sciences version 20 (IBM SPSS Statistics, Armonk, NY, USA, IBM Corp.) was used to analyze the data. Continuous data were summarized as median and range or mean \pm s.D., and the $t$ test was used for significance analysis. Categorical data were expressed as numbers and percentages or ratios and Fisher exact and $\chi^{2}$ tests were used for the significance analysis. A twotailed $P$ value $<0.05$ was considered significant.

\section{Results}

\section{Prevalence of TERT promoter mutations}

The initial characteristics of the TC patients included in this study are summarized in Table 1 . None of the 17 benign multinodular goiters harbored TERT promoter mutations. Overall, C250T and C228T mutations were collectively found in 34 out of 265 cases of TC (12.8\%). This included 10/153 (6.5\%) CPTCs, 8/57 (14.0\%) FVPTCs, 9/30 (30\%) TC-PTCs, $1 / 3$ (30\%) Hurthle cell thyroid cancers (HTCs) 1/5 (20\%) FTCs and 5/13 (38.5\%) PDTCs (Table 1). The C250T mutation was present in only six cases $(2.3 \%)$, including 3/153 CPTC (2\%), 2/57 FVPTC (3.5\%) and 1/30 (3.3\%) TC-PTC (Table 2). The C228T mutation was present in a total of $28 / 265$ (10.6\%) cases, including $7 / 153(4.6 \%)$ CPTC, 6/57 (10.5\%) FVPTC, 8/30 (26.7\%) TC-PTC, 1/3 (33.3\%) HTC, 1/5 (20\%) FTC and 5/13 (38.5\%) PDTC (Table 2). These two mutations were mutually exclusive.

\section{Association of TERT promoter mutations with poor clinicopathological outcomes}

TERT mutations were more common in older ( $\geq 45$ years) than younger patients $(19 / 77(24.7 \%)$ vs $15 / 188(8 \%)$,

Table 1 Characteristics of 265 patients with thyroid cancer

\section{Characteristic}

Median age (range), years

Gender (female:male)

Tumour size, median (range) $\mathrm{cm}$

Tumour multifocality

Extrathyroidal invasion

Vascular Invasion

Lymph node metastasis

Distant metastasis

TNM stage $\mathrm{e}^{\mathrm{a}}$

I

II

III

IV

Radioactive iodine remnant ablation

Radioactive iodine administered activity

Additional therapeutic intervention

Persistent disease at 6-12 months after RAI

Persistent disease at the last follow up

$B R A F^{V 600 E}$ mutation

C228T TERT promotor mutation

C250T TERT promotor mutation

Both TERT promotor mutations

${ }^{\mathrm{a}}$ Data of eight cases were inadequate to allow staging

$P<0.0001$ ) (Table 3). The mean \pm s.D. age was $50.4 \pm 16.7$ years for patients with TERT mutations vs $33.7 \pm 15.7$ years for patients with WT TERT $(P<0.0001)$. Compared to patients with WT TERT, those with TERT promoter mutations showed larger tumor size $(4.75 \pm 2.6 \mathrm{~cm}$ vs $3.2 \pm 2.2 \mathrm{~cm}, P<0.0001)$, more frequent vascular invasion (56.2\% vs $30.8 \%, P=0.009$ ), higher TNM stage (stage III and IV) $(53.3 \%$ vs $15.9 \%, P<0.0001)$ and more common persistent/recurrent disease at 6-12 months after RAI remnant ablation $(71.4 \%$ vs $50.7 \%, P=0.045)$ and at the last follow-up visits (58.1\% vs $34.1 \%, P=0.017$ ) (Table 3). However, TERT promoter mutations were not associated with gender, tumor multifocality, tumor extrathyroidal extension, lymph node metastasis, distant metastasis and the need for additional therapeutic interventions after the initial management (Table 3). The number of foci in the multifocal TC was not different between those with TERT promoter mutations (median 2, range $2-5$ foci) and those with WT TERT (median 3, range 2-4 foci) $(P=0.31)$.

When we restricted the analysis to CPTC only, none of the preceding factors were associated with TERT promoter mutations (Table 4). However, the total number of TERT promoter mutations in CPTC is small $(10 / 153$, $6.5 \%)$, and this lack of association might be due to the small number of cases with positive TERT promoter mutations (Table 2). Similarly, when the analysis was

Published by Bioscientifica Ltd. 
Table 2 Prevalence of TERT promotor and BRAF ${ }^{V 600 E}$ mutations in different subtypes of TC

\begin{tabular}{l} 
Tumor subtype \\
\hline CPTC \\
FVPTC \\
TC-PTC \\
DS-PTC \\
PDTC \\
HTC \\
FTC \\
Total
\end{tabular}

\begin{tabular}{c}
\hline Total no. \\
\hline 153 \\
56 \\
30 \\
4 \\
14 \\
3 \\
5 \\
265
\end{tabular}

\begin{tabular}{c} 
C228T \\
\hline $7(4.6)$ \\
$5(8.9)$ \\
$8(26.7)$ \\
0 \\
$6(42.9)$ \\
$1(33.3)$ \\
$1(20)$ \\
$28(10.6)$
\end{tabular}

\begin{tabular}{l}
\hline C250T \\
\hline $3(2)$ \\
$2(3.6)$ \\
$1(3.3)$ \\
0 \\
0 \\
0 \\
0 \\
$6(2.3)$
\end{tabular}

\begin{tabular}{c}
\hline $\mathbf{C 2 2 8 T}+\mathbf{C 2 5 0 T}$ \\
\hline $10(6.5)$ \\
$7(12.5)$ \\
$9(30)$ \\
0 \\
$6(42.9)$ \\
$1(33.3)$ \\
$1(20)$ \\
$34(12.8)$
\end{tabular}

\begin{tabular}{c}
\hline BRAF $^{\text {VGOOE }}$ \\
\hline $63(41.2)$ \\
$13(23.2)$ \\
$28(93.3)$ \\
$1(25)$ \\
$5(35.7)$ \\
0 \\
0 \\
$110(41.5)$
\end{tabular}

CPTC, conventional papillary thyroid cancer; FVPTC, follicular variant PTC; TC-PTC, tall cell PTC; DS-PTC, diffuse sclerosing type PTC; PDTC, poorly differentiated thyroid cancer; HTC, Hurthle cell thyroid cancer; FTC, follicular thyroid cancer.

limited to FVPTC, no association with any of the preceding factors was noted (Table 4). On the other hand, when the analysis was limited to all other types of TC (excluding CPTC), TERT promoter mutations were associated with age, vascular invasion, high TNM stage, and persistent/recurrent disease at 6-12 months after the initial treatment and at the last follow-up visit (Table 3).

\section{Association of TERT promoter mutation with BRAF ${ }^{V 600 E}$ mutations}

$B R A F^{V 600 E}$ mutation was more common in patients with TERT mutations $(20 / 34,58.8 \%)$ than in those without TERT promoter mutations (90/231, 39\%; $P=0.045)$. Similarly, TERT promoter mutations were more common in patients with $B R A F^{V 600 E}$ mutations $(20 / 110,18.2 \%)$ than in those with WT BRAF $F^{V 600 E}(14 / 155,9 \% ; P=0.045)$. As reported previously, BRAF $F^{V 600 E}$ mutation itself was associated with extrathyroidal invasion, distant metastasis and higher TNM stage (data not shown).

\section{Discussion}

TERT promoter mutations have recently been reported to be common in DTC in patients from North America and Europe (Landa et al. 2013, Liu et al. 2013, Vinagre et al. 2013, Wang et al. 2014a). Studies from these regions have shown that TERT promoter mutations are associated with more aggressive histopathological features and worse outcomes (Landa et al. 2013, Liu et al. 2013, 2014a,b, Vinagre et al. 2013, Liu \& Xing 2014, Melo et al. 2014, Ngeow \& Eng 2014, Wang et al. 2014a, Xing et al. 2014, Gandolfi et al. 2015, Shi et al. 2015). Similar and consistent findings from other ethnic groups would support these conclusions and firmly establish the association between TERT promoter mutations and the risk of aggressiveness of DTC. This was the rationale for undertaking this study. Indeed, we found that TERT promoter mutations were similarly common in our patients and that they were also associated with more aggressive histopathological features and worse outcomes of TC (Tables 2 and 3).

Table 3 The association between TERT promotor mutations and different demographic, histopathological features and TC outcome

\begin{tabular}{l} 
Characteristic \\
\hline Age \\
Sex (M:F) \\
Tumour size (cm) \\
Tumour multifocality \\
Extrathyroidal invasion \\
Vascular invasion \\
Lymph node metastasis \\
Distant metastasis \\
High TNM stage (III and IV) \\
Persistent/recurrent disease at 6-12 months \\
Additional therapeutic interventions \\
Persistent/recurrent disease at last follow up
\end{tabular}

\begin{tabular}{|c|c|c|}
\hline \multicolumn{3}{|c|}{ All types of TC } \\
\hline TERT+ & TERT- & $P$ value \\
\hline $50.4 \pm 16.8$ & $33.7 \pm 15.7$ & $<0.0001$ \\
\hline $11: 23$ & $53: 178$ & 0.33 \\
\hline $4.75 \pm 2.6$ & $3.2 \pm 2.2$ & $<0.0001$ \\
\hline $11 / 34(32.4)$ & $108 / 222(48.6)$ & 0.11 \\
\hline $17 / 33(51.5)$ & $103 / 220(46.8)$ & 0.75 \\
\hline $18 / 32(56.2)$ & $62 / 201(30.8)$ & 0.009 \\
\hline $13 / 23(56.5)$ & $123 / 200(61.5)$ & 0.81 \\
\hline $6 / 33(18.2)$ & $24 / 230(10.4)$ & 0.31 \\
\hline $16 / 30(53.3)$ & $36 / 227$ (15.9) & $<0.0001$ \\
\hline $21 / 29(72.4)$ & $109 / 215(50.7)$ & 0.045 \\
\hline $12 / 31(38.7)$ & $67 / 223(30.0)$ & 0.44 \\
\hline 18/31 (58.1) & $75 / 220(34.1)$ & 0.017 \\
\hline
\end{tabular}

\begin{tabular}{|c|c|c|}
\hline \multicolumn{3}{|c|}{ All types of TC except CPTC } \\
\hline TERT + & TERT - & $P$ value \\
\hline $55.0 \pm 13.6$ & $37.4 \pm 14.9$ & $<0.0001$ \\
\hline $8: 17$ & $24: 78$ & 0.53 \\
\hline $5.2 \pm 2.2$ & $3.6 \pm 2.5$ & 0.005 \\
\hline $8 / 25(32.0)$ & $42 / 100(42.0)$ & 0.49 \\
\hline $13 / 24(54.2)$ & $41 / 98(41.8)$ & 0.39 \\
\hline $15 / 23(65.2)$ & $33 / 94(35.1)$ & 0.017 \\
\hline $8 / 16(50.0)$ & $38 / 85(44.7)$ & 0.91 \\
\hline $6 / 24(25.0)$ & $13 / 101(12.9)$ & 0.24 \\
\hline $14 / 21(66.7)$ & 20/101 (19.8) & $<0.0001$ \\
\hline $17 / 20(85.0)$ & $43 / 92(46.7)$ & 0.004 \\
\hline $10 / 22(45.5)$ & $23 / 96(24.0)$ & 0.08 \\
\hline $15 / 22(68.2)$ & $29 / 96(30.2)$ & 0.002 \\
\hline
\end{tabular}


Table 4 The association between TERT promotor mutations and different demographic, histopathological features and outcome of CPTC and FVPTC

\begin{tabular}{l} 
Characteristic \\
\hline Age \\
Sex (M:F) \\
Tumour size (cm) \\
Tumour multifocality \\
Extrathyroidal invasion \\
Vascular invasion \\
Lymph node metastasis \\
Distant metastasis \\
High TNM stage (III and IV) \\
Persistent/recurrent disease at 6-12 months \\
Additional therapeutic interventions \\
Persistent/recurrent disease at last follow up \\
\hline
\end{tabular}

\begin{tabular}{c}
\hline TERT+ \\
\hline $37.4 \pm 18.7$ \\
$3: 6$ \\
$3.7 \pm 3.5$ \\
$3 / 9(33.3)$ \\
$4 / 9(44.4)$ \\
$3 / 9(33.3)$ \\
$5 / 7(71.4)$ \\
$0 / 9(0)$ \\
$2 / 9(22.2)$ \\
$4 / 9(44.4)$ \\
$12 / 31(38.7)$ \\
$2 / 9(22.2)$ \\
\hline
\end{tabular}

CPTC only

\begin{tabular}{|c|c|}
\hline TERT- & $P$ value \\
\hline $30.8 \pm 15.7$ & 0.23 \\
\hline 29:100 & 0.74 \\
\hline $2.8 \pm 1.8$ & 0.20 \\
\hline $6 / 122(54.1)$ & 0.39 \\
\hline $2 / 122(50.8)$ & 0.98 \\
\hline 9/107 (27.1) & 0.99 \\
\hline 5/115 (73.9) & 1.0 \\
\hline $1 / 129(8.5)$ & 1.0 \\
\hline $6 / 126$ (12.7) & 0.34 \\
\hline $6 / 123$ (53.7) & 0.73 \\
\hline $7 / 223(30.0)$ & 0.44 \\
\hline 4/127 (34.6) & 0.72 \\
\hline
\end{tabular}

\begin{tabular}{|c|c|c|}
\hline \multicolumn{3}{|c|}{ FVPTC only } \\
\hline TERT + & TERT - & $P$ value \\
\hline $49.0 \pm 14.6$ & $37.7 \pm 14.5$ & 0.059 \\
\hline $1: 6$ & $9: 40$ & 1.0 \\
\hline $4.9 \pm 2.9$ & $3.5 \pm 2.5$ & 0.21 \\
\hline $2 / 7(28.6)$ & $19 / 48(39.6)$ & 0.70 \\
\hline $1 / 7(14.3)$ & $13 / 48(27.1)$ & 0.66 \\
\hline $4 / 7(57.1)$ & $10 / 46(21.7)$ & 0.07 \\
\hline $2 / 5(40.0)$ & $10 / 38(26.3)$ & 0.61 \\
\hline $1 / 7(14.3)$ & $6 / 48(12.5)$ & 1.0 \\
\hline $3 / 7(42.9)$ & $8 / 48$ (16.7) & 0.13 \\
\hline $2 / 5(40.0)$ & $19 / 45(42.2)$ & 1.0 \\
\hline $0 / 6(0)$ & 8/47 (17.0) & 0.57 \\
\hline $2 / 6(33.3)$ & $13 / 46(28.3)$ & 1.0 \\
\hline
\end{tabular}

TERT promoter mutations were initially found to occur in melanoma, CNS and bladder tumors (Horn et al. 2013, Huang et al. 2013, Vinagre et al. 2013). Previous studies screening different types of tumors for TERT promoter mutations have shown that the rates of these mutations were 59, 43, 29 and 10\% in bladder, CNS, melanoma and thyroid tumors respectively (Vinagre et al. 2013). Liu et al. (2013) studied TERT promoter mutations in different subtypes of TCs and thyroid cell lines and found that the two mutations occurred in 13.9, 46.3 and $91.7 \%$ of the samples of FTC, ATC and thyroid cell lines respectively. C250T mutation was much less common, but both mutations were mutually exclusive. The C228T mutation was found in $12.3 \%$ of CPTCs, $3.6 \%$ of FVPTCs, $30.8 \%$ of TC-PTC samples and in none of 16 medullary TC samples. $B R A F^{V 600 E}$ mutation was more frequent in cases with C228T mutations than in those without mutation. Several subsequent studies from North America and Europe showed that TERT promoter mutations occurred in about 10-15\% TCs (Landa et al. 2013, Vinagre et al. 2013, 2014, Fredriksson et al. 2014, Liu et al. 2014a, Melo et al. 2014, Wang et al. 2014a,b, Gandolfi et al. 2015, Muzza et al. 2015, Shi et al. 2015). These mutations were more common in the poorly differentiated and anaplastic TC and were associated with more aggressive features of DTC (Landa et al. 2013, Liu et al. 2013). Further studies showed a synergistic prognostic effect of these mutations with the $B R A F^{V 600 E}$ mutation - a well-known mutation in DTC with worse prognostic features (Xing et al. 2014).

In our study, TERT promoter mutations were not found in any of the 17 benign thyroid tumors. This is consistent with previous studies. In six previous studies that included 476 benign thyroid tumors of different types, none was found to harbor TERT promoter mutations (Liu et al. 2013, 2014a, Vinagre et al. 2013, Liu \& Xing 2014, Melo et al. 2014, Muzza et al. 2015). However, in one study, one out of 58 benign follicular adenomas and three of 18 atypical follicular adenomas harbored C228T TERT promoter mutation, suggesting that this mutation is an early molecular event in thyroid tumorigenesis (Wang et al. 2014a). This finding has not been substantiated in any other study, including our study. CPTC is the most common type of TC. In our study, the prevalence of TERT promoter mutations in CPTC was relatively low (6.5\%). Other studies showed rates that ranged between 4.5 and $22.5 \%$, with most studies showing a frequency of TERT promoter mutations of around $12 \%$ in CPTC (Landa et al. 2013, Liu et al. 2013, 2014a, Vinagre et al. 2013, Liu \& Xing 2014, Melo et al. 2014, Xing et al. 2014, Gandolfi et al. 2015, Muzza et al. 2015). Similar to previous studies, ours showed TERT promoter mutations in $8 / 57$ (14\%) cases of FVPTC. This is similar to the rate reported in a large series of TC in which 18 out of 103 cases (14.6\%) of FVPTC harbored TERT promoter mutations (Xing et al. 2014) and another smaller study from Europe showing a similar rate (Gandolfi et al. 2015).

In our study, the rate of TERT promoter mutations was significantly higher in other subtypes of DTC compared to those in CPTC and FVPTC. TERT promoter mutations were found in $30 \%$ of TC-PTC, 30\% HTC, $20 \%$ FTC and $38.5 \%$ PDTC (Table 2). Previous studies showed rates of TERT promoter mutations varying between 13.9 and $36.4 \%$ in FTC with most studies showing rates of around 14-17\% (Landa et al. 2013, Liu et al. 2013, 2014b, Vinagre et al. 2013, Melo et al. 2014, Shi et al. 2015). In one study that included only HTC, TERT promoter mutations were

Published by Bioscientifica Ltd. 
screened in a subset of 61 cases, of which 8 (13.1\%) harbored C228T mutation (Chindris et al. 2015). In another study that included 25 cases of HTC, 4 (16\%) of them harbored TERT promoter mutations (Landa et al. 2013). The rates of TERT promoter mutations varied between 21 and 51.7\% in PDTC (Landa et al. 2013, Liu et al. 2013, 2014b, Vinagre et al. 2013, Melo et al. 2014) and between 13 and 50\% in ATC (Landa et al. 2013, Liu et al. 2013, 2014b, Vinagre et al. 2013, Melo et al. 2014, Shi et al. 2015).

The association between TERT promoter mutations and aggressive features of TC has been demonstrated in most previous studies (Landa et al. 2013, Liu et al. 2013, 2014a, Melo et al. 2014, Liu \& Xing 2014, Xing et al. 2014, Gandolfi et al. 2015, Shi et al. 2015). However, the relative significance of each of those features varies among these studies. A consistent finding is that TERT promoter mutations occur more frequently at an older age and are associated with distant metastasis, higher tumor stage and poorly differentiated types of TC (Vinagre et al. 2013, Liu et al. 2014a, Melo et al. 2014, Wang et al. 2014a, Gandolfi et al. 2015, Muzza et al. 2015, Shi et al. 2015). Interestingly, tumor size, extrathyroidal invasion, tumor multifocality, lymph node metastasis and vascular invasion are not consistently associated with TERT promoter mutations. These conflicting findings between different studies might be due to the differences in characterizing the patients studied, sample size and proportions of different subtypes of TC included. In our study, overall, there was a strong association with patient's age and tumor size, vascular invasion and short- and long-term persistent/recurrence rates. However, there was no association with extrathyroidal extension, tumor multifocality, lymph node and distant metastasis. Interestingly, when we limited the analysis to CPTC or FVPTC, no association with any of these factors was noted (Table 4). In contrast, a stronger association was seen between these factors and TERT promoter mutations in non-CPTC types (Table 3 ). This suggests that TERT promoter mutations are more characteristic of high-grade TC and that they may have a particularly useful prognostic value in these subtypes of TC. This idea is consistent with the fact that previous studies showed a much higher rate of TERT promoter mutations in high-grade non-CPTC subtypes of TC (Landa et al. 2013, Liu et al. 2013, Xing et al. 2014).

The association between $B R A F^{V 600 E}$ and TERT promoter mutations has also been demonstrated in most but not all studies (Liu et al. 2014a, Melo et al. 2014, Xing et al. 2014, Gandolfi et al. 2015, Muzza et al. 2015, Shi et al. 2015). Tumors with $B R A F^{V 600 E}$ mutations are more likely to harbor TERT promoter mutations and vice versa (Liu et al. 2014a, Melo et al. 2014, Xing et al. 2014, Shi et al. 2015). In our study, we have also shown a similar strong association between $B R A F^{V 600 E}$ and TERT promoter mutations. Previous studies have also shown a synergistic prognostic effect of $B R A F^{V 600 E}$ and TERT promoter mutations with significantly more aggressive features and higher risk of recurrence in tumors harboring both mutations compared with those with either one or none of them (Xing et al. 2014). Other investigations have shown that TERT promoter mutations are stronger prognostically than $B R A F^{V 600 E}$ mutation, but found no difference in recurrence rates between those tumors that carry both or either one of the mutations (Muzza et al. 2015). These studies, however, were relatively small, with a limited number of cases harbouring both mutations. For similar reason, we did not find synergy between TERT promoter and $B R A F^{V 600 E}$ mutations in the present study. These conflicting results might also reflect the role of tumor microenvironment. Recent studies have shown a role for $B R A F^{V 6 O O E}$ mutation in thyroid tumors that are mesenchymal in origin (Sadow et al. 2014). Sadow et al. reported the presence of $B R A F^{V 600 E}$ mutation in three benign myopericytomas, a rare frequently benign mesenchymal tumor. One of these three myopericytomas was in the thyroid gland. Myopericytomas that harboured BRAF V600E mutation were likely to be multifocal, invasive and recurrent. In vitro and in vivo studies showed significant effects of $B R A F^{V 600 E}$ mutation on the tumor microenvironment promoting invasiveness and angiogenesis. These effects were reversible by the specific anti BRAF inhibitor, Vemurafenib, in tumors harbouring $B R A F^{V 600 E}$ mutation but not in the WT BRAF tumors (Sadow et al. 2014). This suggests that cells other than the epithelial follicular cells from which TC normally arises might harbour mutations that contribute to the progression of cancer. Similar effects and interactions of TERT promoter mutations with tumor microenvironment are possible including their presence in the mesenchymal cells. The study of these mutations in cells from the tumor microenvironment can be facilitated by using orthotopic mouse models of TC (Antonello \& Nucera 2014).

In summary, our study shows that TERT promoter mutations are consistently detectable in a significant percentage of TC patients from a different ethnic group. The rate of these mutations observed in our study is similar to that in other ethnic populations. TERT promoter mutations are more likely to occur in tumors from older patients, in larger and less differentiated tumors and in association with more aggressive tumor features. The

Published by Bioscientifica Ltd 
findings of our study confirmed the findings in other ethnic groups and therefore strongly support a general important pathogenetic role of TERT promoter mutations in TC. Our findings also highlight the significant prognostic value of TERT promoter mutations for the aggressiveness of TC. To our knowledge, this is the first study reporting TERT promoter mutations and their association with the aggressiveness of TC in a large cohort of patients with TC from the Middle East, supporting the inter-ethnic consistency of the previous findings on these mutations.

\section{Declaration of interest}

The authors declare that there is no conflict of interest that could be perceived as prejudicing the impartiality of the research reported.

\section{Funding}

This research did not receive any specific grant from any funding agency in the public, commercial or not-for-profit sector.

\section{References}

Agrawal N, Akbani R, Aksoy BA, Ally A, Arachchi H, Asa SL, Auman JT, Balasundaram M, Balu S, Baylin SB et al. 2014 Integrated genomic characterization of papillary thyroid carcinoma. Cell 159 676-690. (doi:10.1016/j.cell.2014.09.050)

Antonello ZA \& Nucera C 2014 Orthotopic mouse models for the preclinical and translational study of targeted therapies against metastatic human thyroid carcinoma with BRAF(V600E) or wild-type BRAF. Oncogene 33 5397-5404. (doi:10.1038/onc.2013.544)

Blackburn EH 2005 Telomeres and telomerase: their mechanisms of action and the effects of altering their functions. FEBS Letters $\mathbf{5 7 9} 859-862$. (doi:10.1016/j.febslet.2004.11.036)

Chindris AM, Casler JD, Bernet VJ, Rivera M, Thomas C, Kachergus JM, Necela BM, Hay ID, Westphal SA, Grant CS et al. 2015 Clinical and molecular features of Hurthle cell carcinoma of the thyroid. Journal of Clinical Endocrinology and Metabolism 100 55-62. (doi:10.1210/jc.2014-1634)

Enewold L, Zhu K, Ron E, Marrogi AJ, Stojadinovic A, Peoples GE \& Devesa SS 2009 Rising thyroid cancer incidence in the United States by demographic and tumor characteristics, 1980-2005. Cancer Epidemiology, Biomarkers \& Prevention 18 784-791. (doi:10.1158/ 1055-9965.EPI-08-0960)

Fredriksson NJ, Ny L, Nilsson JA \& Larsson E 2014 Systematic analysis of noncoding somatic mutations and gene expression alterations across 14 tumor types. Nature Genetics 46 1258-1263. (doi:10.1038/ ng.3141)

Gandolfi G, Ragazzi M, Frasoldati A, Piana S, Ciarrocchi A \& Sancisi V 2015 TERT promoter mutations are associated with distant metastases in papillary thyroid carcinoma. European Journal of Endocrinology/European Federation of Endocrine Societies 172 403-413. (doi:10.1530/EJE-14-0837)

Horn S, Figl A, Rachakonda PS, Fischer C, Sucker A, Gast A, Kadel S, Moll I, Nagore E, Hemminki K et al. 2013 TERT promoter mutations in familial and sporadic melanoma. Science 339 959-961. (doi:10.1126/science. 1230062)
Huang FW, Hodis E, Xu MJ, Kryukov GV, Chin L \& Garraway LA 2013 Highly recurrent TERT promoter mutations in human melanoma. Science 339 957-959. (doi:10.1126/science.1229259)

Jemal A, Bray F, Center MM, Ferlay J, Ward E \& Forman D 2011 Global cancer statistics. CA: A Cancer Journal for Clinicians 61 69-90. (doi:10.3322/caac.20107)

Jemal A, Simard EP, Dorell C, Noone AM, Markowitz LE, Kohler B, Eheman C, Saraiya M, Bandi P, Saslow D et al. 2013 Annual Report to the Nation on the Status of Cancer, 1975-2009, featuring the burden and trends in human papillomavirus (HPV)-associated cancers and HPV vaccination coverage levels. Journal of the National Cancer Institute $\mathbf{1 0 5}$ 175-201. (doi:10.1093/jnci/djs491)

Jung CK, Little MP, Lubin JH, Brenner AV, Wells SA Jr, Sigurdson AJ \& Nikiforov YE 2014 The increase in thyroid cancer incidence during the last four decades is accompanied by a high frequency of BRAF mutations and a sharp increase in RAS mutations. Journal of Clinical Endocrinology and Metabolism 99 E276-E285. (doi:10.1210/ jc.2013-2503)

Landa I, Ganly I, Chan TA, Mitsutake N, Matsuse M, Ibrahimpasic T, Ghossein RA \& Fagin JA 2013 Frequent somatic TERT promoter mutations in thyroid cancer: higher prevalence in advanced forms of the disease. Journal of Clinical Endocrinology and Metabolism 98 E1562-E1566. (doi:10.1210/jc.2013-2383)

Liu R \& Xing M 2014 Diagnostic and prognostic TERT promoter mutations in thyroid fine-needle aspiration biopsy. Endocrine-Related Cancer 21 825-830. (doi:10.1530/ERC-14-0359)

Liu X, Bishop J, Shan Y, Pai S, Liu D, Murugan AK, Sun H, El-Naggar AK \& Xing M 2013 Highly prevalent TERT promoter mutations in aggressive thyroid cancers. Endocrine-Related Cancer 20 603-610. (doi:10.1530/ ERC-13-0210)

Liu X, Qu S, Liu R, Sheng C, Shi X, Zhu G, Murugan AK, Guan H, Yu H, Wang Y et al. 2014a TERT promoter mutations and their association with BRAF V600E mutation and aggressive clinicopathological characteristics of thyroid cancer. Journal of Clinical Endocrinology and Metabolism 99 E1130-E1136. (doi:10.1210/jc.2013-4048)

Liu T, Wang N, Cao J, Sofiadis A, Dinets A, Zedenius J, Larsson C \& Xu D $2014 b$ The age- and shorter telomere-dependent TERT promoter mutation in follicular thyroid cell-derived carcinomas. Oncogene $\mathbf{3 3}$ 4978-4984. (doi:10.1038/onc.2013.446)

Melo M, da Rocha AG, Vinagre J, Batista R, Peixoto J, Tavares C, Celestino R, Almeida A, Salgado C, Eloy C et al. 2014 TERT promoter mutations are a major indicator of poor outcome in differentiated thyroid carcinomas. Journal of Clinical Endocrinology and Metabolism 99 E754-E765. (doi:10.1210/jc.2013-3734)

Mocellin S, Pooley KA \& Nitti D 2013 Telomerase and the search for the end of cancer. Trends in Molecular Medicine 19 125-133. (doi:10.1016/ j.molmed.2012.11.006)

Muzza M, Colombo C, Rossi S, Tosi D, Cirello V, Perrino M, De Leo S, Magnani E, Pignatti E, Vigo B et al. 2015 Telomerase in differentiated thyroid cancer: promoter mutations, expression and localization. Molecular and Cellular Endocrinology 399 288-295. (doi:10.1016/j.mce. 2014.10.019)

Ngeow J \& Eng C 2014 TERT and BRAF in thyroid cancer: teaming up for trouble. Journal of Clinical Oncology 32 2683-2684. (doi:10.1200/JCO. 2014.56.5614)

Sadow PM, Priolo C, Nanni S, Karreth FA, Duquette M, Martinelli R, Husain A, Clohessy J, Kutzner H, Mentzel T et al. 2014 Role of BRAFV600E in the first preclinical model of multifocal infiltrating myopericytoma development and microenvironment. Journal of the National Cancer Institute 106 dju182. (doi:10.1093/jnci/dju182)

Shi X, Liu R, Qu S, Zhu G, Bishop J, Liu X, Sun H, Shan Z, Wang E, Luo Y et al. 2015 Association of TERT promoter mutation 1,295,228 C > T with BRAF V600E mutation, older patient age, and distant metastasis in anaplastic thyroid cancer. Journal of Clinical Endocrinology and Metabolism 100 E632-E637. (doi:10.1210/jc.2014-3606) 
Smekalova EM, Shubernetskaya OS, Zvereva MI, Gromenko EV, Rubtsova MP \& Dontsova OA 2012 Telomerase RNA biosynthesis and processing. Biochemistry 77 1120-1128.

Vinagre J, Almeida A, Populo H, Batista R, Lyra J, Pinto V, Coelho R, Celestino R, Prazeres H, Lima L et al. 2013 Frequency of TERT promoter mutations in human cancers. Nature Communications 42185. (doi:10.1038/ncomms3185)

Vinagre J, Pinto V, Celestino R, Reis M, Populo H, Boaventura P, Melo M, Catarino T, Lima J, Lopes JM et al. 2014 Telomerase promoter mutations in cancer: an emerging molecular biomarker? Virchows Archiv 465 119-133. (doi:10.1007/s00428-014-1608-4)

Wang N, Liu T, Sofiadis A, Juhlin CC, Zedenius J, Hoog A, Larsson C \& Xu D $2014 a$ TERT promoter mutation as an early genetic event activating telomerase in follicular thyroid adenoma (FTA) and atypical FTA. Cancer 120 2965-2979. (doi:10.1002/cncr.28800)

Wang N, Xu D, Sofiadis A, Hoog A, Vukojevic V, Backdahl M, Zedenius J \& Larsson C 2014b Telomerase-dependent and independent telomere maintenance and its clinical implications in medullary thyroid carcinoma. Journal of Clinical Endocrinology and Metabolism 99 E1571-E1579. (doi:10.1210/jc.2014-1158)

Xing M 2013 Molecular pathogenesis and mechanisms of thyroid cancer. Nature Reviews. Cancer 13 184-199. (doi:10.1038/nrc3431)

Xing M, Liu R, Liu X, Murugan AK, Zhu G, Zeiger MA, Pai S \& Bishop J 2014 BRAF V600E and TERT promoter mutations cooperatively identify the most aggressive papillary thyroid cancer with highest recurrence. Journal of Clinical Oncology 32 2718-2726. (doi:10.1200/JCO.2014.55.5094)

Received in final form 7 September 2015

Accepted 9 September 2015

Made available online as an Accepted Preprint

9 September 2015
Published by Bioscientifica Ltd. 